\title{
ARTICLE OPEN Health status measured by the Clinical COPD Questionnaire (CCQ) improves following post-acute pulmonary rehabilitation in patients with advanced COPD: a prospective observational study
}

\author{
Eléonore F van Dam van Isselt ${ }^{1,2}$, Monica Spruit ${ }^{1}$, Karin H Groenewegen-Sipkema ${ }^{3}$, Niels H Chavannes ${ }^{1}$ and Wilco P Achterberg ${ }^{1}$
}

\begin{abstract}
AIMS: To evaluate outcomes of the Clinical Chronic obstructive pulmonary disease (COPD) Questionnaire (CCQ) in patients with advanced COPD admitted for a post-acute pulmonary rehabilitation (PR) programme and to relate (change in) health status to lung function, degree of dyspnoea and (change in) functional capacity.

METHODS: This is a prospective observational study in patients with advanced COPD admitted for a post-acute PR programme in a skilled nursing facility. Health status (CCQ) and functional capacity were measured before and after rehabilitation.

RESULTS: Health status measured by the CCQ was severely impaired and showed significant and clinically relevant improvement during the post-acute PR programme. Moderate to strong correlations were found between CCQ scores and functional capacity on admission and at discharge. Moderate correlations were found between improvement in CCQ scores and improvement in functional capacity. No correlation was found between CCQ scores and lung function (forced expiratory volume in $1 \mathrm{~s} \%$ predicted). CONCLUSIONS: Health status measured by the CCQ improves following a post-acute PR programme in patients with advanced COPD and correlates with improvement in functional capacity. These results suggest that the CCQ is sensitive to change in response to $P R$ in this specific group of patients.
\end{abstract}

npj Primary Care Respiratory Medicine (2014) 24, Article number: 14007; doi:10.1038/npjpcrm.2014.7; published online 20 May 2014

\section{INTRODUCTION}

In chronic obstructive pulmonary disease (COPD), health-related quality of life (HRQoL) is increasingly recognised as an important measurement that reflects the patient's perspective of the impact of the disease on symptom burden, functional capacity and psychosocial functioning. ${ }^{1,2}$ Therefore, more traditional parameters of disease severity (such as lung function) should be supplemented with measurements of HRQoL or health status. However, instruments to assess HRQoL or health status are often time-consuming and/or relatively difficult for the patients to comprehend. Furthermore, although instruments to measure HRQoL are widely used as important outcome measurements in research, their use in daily practice (especially in primary care) is limited.

In 2003 van der Molen et al..$^{3}$ developed and validated the Clinical COPD Questionnaire (CCQ). The CCQ is a simple and reliable 10-item tool that focuses not only on the clinical status of the airways, but also on functional limitations and psychosocial dysfunction. The CCQ consists of three separate domains (i.e., symptoms, functional state and mental state) and was originally developed to measure the clinical health status in patients with COPD. Treatment in clinical practice can be aimed at these subdomains, which elaborates on tailor-made medicine in patients with COPD. The CCQ can also be used to evaluate the adequacy of clinical management ${ }^{4}$ and to assess functional performance. ${ }^{5}$ In fact, the functional state domain of the CCQ is reported to be the best patient-reported outcome tool for assessing functional performance in patients with COPD in primary care. ${ }^{6}$ Furthermore, the CCQ can be used to measure the effect of integrated disease management interventions in primary $\mathrm{care}^{6}$ and to predict exacerbations ${ }^{7}$ and mortality ${ }^{2}$ in patients with mild to moderate COPD. However, little is known about the use of the CCQ in patients with advanced COPD, or its use as a primary outcome measure in pulmonary rehabilitation (PR).

Therefore, the aim of the present study was to evaluate outcomes of the CCQ in patients with advanced COPD admitted for post-acute PR and to correlate (improvement in) health status measured by the CCQ to lung function, degree of dyspnoea and (improvement in) functional capacity.

\section{MATERIALS AND METHODS}

Design and setting

This is a prospective observational study designed to evaluate outcomes of the CCQ in patients with advanced COPD and to relate (improvement in) health status to lung function, degree of dyspnoea and (improvement in) functional capacity. The study was conducted at a skilled nursing facility (SNF) that offers geriatric rehabilitation for patients with advanced COPD. Data were collected from the patients' files by the patients' physicians and transferred to an anonymous data file (SPPS 20). Given the fact that this observational study measured a form of structured usual care, no written informed consent was required.

Baseline measurements (TO) were collected and performed within 3 days after admission to the SNF; these consisted of patient and disease characteristics, health status (CCQ), degree of dyspnoea (modified Medical Research Council (mMRC) scale) and functional capacity (Barthel Index (BI) and the Six-Minute Walking Test (6MWT)). At discharge from the SNF (T1),

${ }^{1}$ Department of Public Health and Primary Care, Leiden University Medical Center, Leiden, The Netherlands; ${ }^{2}$ Zorggroep Solis, Deventer, The Netherlands and ${ }^{3}$ Pulmonary Department, Deventer Hospital, Deventer, The Netherlands.

Correspondence: EF van Dam van Isselt (E.F.vanDamvanlsselt@lumc.nl)

Received 29 October 2013; revised 27 January 2014; accepted 9 February 2014 
health status and functional capacity were measured again. Data were collected from May 2009 until January 2011.

\section{Participants}

Patients with severe (Global Initiative for Chronic Obstructive Lung Disease $(G O L D)^{8}$ stage 3) to very severe (GOLD stage 4) COPD, admitted to the hospital for an acute exacerbation, were indicated for the PR programme by a pulmonologist. PR was considered appropriate when patients suffered from high symptom burden and/or a substantial decline in health status and functional capacity without sufficient recovery during hospital stay. Furthermore, a multidisciplinary approach was required to achieve improvement in health status instead of physical therapy alone. Patients who lacked motivation or patients with prominent psychiatric or cognitive dysfunction interfering with PR were excluded from the programme. All patients admitted to the PR programme were eligible to participate in this study.

\section{Pulmonary rehabilitation programme}

The PR programme was offered at an SNF that offers geriatric rehabilitation. Geriatric rehabilitation consists of post-acute restorative inpatient treatment with a multidisciplinary patient-centred approach in a therapeutic environment. ${ }^{9}$ Geriatric rehabilitation does not differ from rehabilitation medicine in its approach. However, patients admitted to geriatric rehabilitation programmes do have different characteristics: higher age, substantial comorbidity and limited functional and training capacity. ${ }^{10}$ The SNF at which the present study was conducted has one ward with a multidisciplinary team that is specialised in post-acute care and rehabilitation for patients with advanced COPD. ${ }^{11}$ The PR programme contains several modules on different aspects of rehabilitation. Goal setting and duration of the programme is tailored to the individual patient, and the programme is evaluated weekly and adjusted (as needed) by the multidisciplinary team. All patients follow a standardised weekly programme that contains a minimum of five 40 -min physiotherapy sessions, occupational therapy once or twice a week, analysis and evaluation of nutritional status every week and weekly group sessions (education of patients and relatives, and peer support contact). Assignment to therapies is stringent. A detailed description of the PR programme was recently published. ${ }^{11}$

Health status and degree of dyspnoea

Health status was measured using the Dutch version of the CCQ. ${ }^{3}$ The CCQ is a validated and reliable 10-item, self-administered questionnaire. The CCQ consists of three subdomains: symptoms, functional state and mental state. Items are scored on a Likert scale (range 0-60). The final score is the sum of all items divided by 10 ; separate scores for all three domains can be calculated. Higher scores indicate a worse health status. The minimal clinically important difference (MCID) of the CCQ total score is $-0.4 .^{12}$ Degree of dyspnoea was measured using the mMRC dyspnoea scale. ${ }^{7}$ The mMRC is an ordinal four-point scale (grades 0-4) based on degrees of various physical activities that precipitate dyspnoea. Grade 4 represents the most severe category.

\section{Functional capacity}

Functional capacity was measured by the modified $\mathrm{BI}$ and the 6MWT. The $\mathrm{BI}$ measures activities of daily living and is a valid, reliable and widely used instrument to assess activities of daily living improvement during rehabilitation programmes. ${ }^{13}$ The total score ranges from 0 to 20 , with 20 representing complete functional independence. The MCID for the $\mathrm{BI}$ is not well established for COPD patients. In stroke patients the MCID of the $\mathrm{BI}$ was calculated at $+1.85 .^{14}$ The $\mathrm{BI}$ was assessed by a specialised nurse of the SNF.

The 6MWT is a practical, easy-to-perform and widely used instrument for measuring exercise capacity in patients with COPD. The 6MWT is strongly predictive of survival in patients with COPD and an important outcome measure for PR. ${ }^{15,16}$ The MCID for the 6MWT in patients with severe COPD is $+26( \pm 2) \mathrm{m}^{17}$ The 6MWT was assessed by a physiotherapist of the multidisciplinary team of the SNF in a standardised setting in accordance with international guidelines. ${ }^{15}$

\section{Statistical analysis}

All data were processed using SPSS (IBM SPSS Statistics for Windows, Version 20.0, IBM, Armonk, NY, USA). Descriptive analyses were used for measurements on admission (T0) and at discharge (T1). To compare the mean outcome measurements on admission (T0) and discharge (T1), the paired sample $t$-test was used. In case of skewed data (BI), the nonparametric Wilcoxon test was used. To investigate potential regression to the mean, a linear regression analysis was performed for change (T1 - T0) against baseline measurements (T0). Pearson's correlation coefficient was calculated to determine the strength of linear correlations between pairs of variables of interest. In case of skewed data or measurements at interval level, Spearman's correlation coefficient was calculated. We defined statistical significance at $P \leq 0.05$ (two-sided level of significance).

\section{RESULTS}

Study population

A total of 63 patients entered the programme during the specified period and were eligible to participate in this study. Of them, two were excluded because of a different diagnosis (one for asthma and one for small airway disease), two (5\%) died during the rehabilitation programme and one dropped out because of lack of motivation.

Patient and disease characteristics are presented in Table 1. Mean length of admission to the SNF was $44.1( \pm 30.2)$ days. The study population consisted of 30 women and 31 men with a mean age of $68.9( \pm 9.9)$ years. All patients had advanced COPD (GOLD stage 3 or 4) with a mean forced expiratory volume in $1 \mathrm{~s}$ $\left(\mathrm{FEV}_{1}\right) \%$ predicted of $31.6( \pm 10.8)$; in addition, 17 patients $(28 \%)$ were on long-term oxygen therapy.

Health status, degree of dyspnoea and functional capacity on admission

On admission, the mean CCQ score was $3.5( \pm 0.9)$, indicating severely limited health status, and the mean score on the mMRC was $3.8( \pm 1.1)$. Functional capacity was limited, as the median BI score (17 (interquartile range 15-18)) indicated care dependency and the mean 6MWT $(208( \pm 119) \mathrm{m})$ indicated limited exercise capacity.

Course of health status and functional capacity during the PR programme

During the PR programme there was a significant and clinically relevant improvement in health status (CCQ) and functiona capacity (BI and 6MWT) (Table 2). On admission the mean CCQ was 3.5 ( \pm 0.9 , range $1.3-5.8)$ and was $2.2( \pm 1.0$, range $0.6-4.4)$ at discharge. All three subdomains of the CCQ showed significant improvement: of all patients, $86.8 \%$ showed an improvement on the CCQ equal to the MCID or more; in two patients the CCQ score

Table 1. Patient and disease characteristics on admission (baseline/TO)

No. of patients

Age in years (s.d.)

Sex: woman, $n(\%)$

Sex: woman, $n(\%)$

LOA-H in days (IQR)

LOA-SNF in days (IQR)

GOLD 3, $n(\%)$

GOLD 4, $n$ (\%)

$\mathrm{FEV}_{1} \%$ of predicted (s.d.)

LTOT, $n$ (\%)

Smoker, $n$ (\%)

30 (49)

28 (46)

$8(7-11)$

$35(21-61)$

$29(48)$

32 (52)

$32.9(10.8)$

17 (28)

$10(16.5)$

Categorical variables are described as frequencies, while continuous variables were tested for normality and are presented as mean (s.d.) or median (IQR) in case of skewed data.

Abbreviations: $\mathrm{FEV}_{1}$, forced expiratory volume in $1 \mathrm{~s}$; GOLD, Global Initiative for Chronic Obstructive Lung Disease; IQR, interquartile range LOA-H, length of admission during hospital stay; LOA-SNF, length of admission during rehabilitation; LTOT, long-term oxygen therapy. 
Table 2. Outcomes of measurements on admission (TO) and discharge $(\mathrm{T} 1)$

\begin{tabular}{|c|c|c|c|c|c|}
\hline & & TO & & $T 1$ & $P$ \\
\hline CCQ (s.d.) & 60 & $3.5(0.9)$ & 53 & $2.2(1.0)$ & $<0.001^{\mathrm{a}}$ \\
\hline Symptoms & 60 & $3.7(1.1)$ & 54 & $2.4(1.1)$ & $<0.001^{\mathrm{a}}$ \\
\hline Functional state & 60 & $3.9(1.2)$ & 53 & $2.6(1.4)$ & $<0.001^{\mathrm{a}}$ \\
\hline Mental state & 60 & $2.3(1.6)$ & 53 & $1.3(1.4)$ & $<0.001^{a}$ \\
\hline Barthel Index (IQR) & 61 & $17(15-18)$ & 58 & $20(17-20)$ & $<0.001^{\mathrm{b}}$ \\
\hline 6MWT, metres (s.d.) & 58 & 208 (119) & 54 & $274(122)$ & $<0.001^{\mathrm{a}}$ \\
\hline \multicolumn{6}{|c|}{$\begin{array}{l}\text { Variables were tested for normality and are presented as mean (s.d.) or } \\
\text { median (IQR) in case of skewed data. } \\
\text { Abbreviations: } 6 \mathrm{MWT} \text {, Six-Minute Walking Test; IQR, interquartile range. } \\
\text { a'Variables were compared between T0 and T1 using a paired sample } t \text {-test, } \\
\text { as appropriate. } \\
\text { bVariables were compared between T0 and T1 using a paired sample } \\
\text { Wilcoxon test, as appropriate. }\end{array}$} \\
\hline
\end{tabular}

did not change; and in three patients the score increased, indicating a deterioration of health status during the programme. On admission the median BI score was 17 (range 5-20) and was 20 (range 11-20) at discharge. In 41 patients the $\mathrm{Bl}$ improved two points or more, in 2 patients the $\mathrm{Bl}$ at discharge was lower than on admission and in 9 patients the $\mathrm{Bl}$ did not change during the programme. However, all these latter patients had a maximum score (19 or 20) on admission. The mean 6MWT improved from $208 \mathrm{~m}$ (range 36-568 m) on admission to $274 \mathrm{~m}$ (range 61-634 m) at discharge. In $71.7 \%$ of the patients the $6 \mathrm{MWT}$ improved $>26 \mathrm{~m}$. In two patients the 6MWT did not change and in six patients the 6MWT decreased.

To investigate potential for regression to the mean, a linear regression analysis was performed for change (T1-T0) against baseline measurements (T0). Figure 1 shows the relation between improvement in CCQ (CCQ-delta) and CCQ at baseline (CCQ-T0). Results from the linear regression model show a Y-intercept $\left(b_{0}\right)$ of 0.36 and a gradient of the regression line $\left(b_{1}\right)$, representing the change in outcome variable (CCQ-delta) associated with one-unit change in the predictor (CCQ-T0) of -0.473 .

Relationship between health status, lung function, functional capacity and degree of dyspnoea on admission and discharge To determine the correlation between health status as measured by the CCQ and lung function ( $\mathrm{FEV}_{1} \%$ pred), functional capacity (6MWT and $\mathrm{BI}$ ) and degree of dyspnoea (mMRC) on admission and at discharge, we calculated the correlation coefficients between these variables (Table 3). On admission, there was a moderate to strong correlation between CCQ (CCQ total score and CCQ function domain score) and functional capacity measured by the 6MWT (CCQ total score: $r=-0.400, P=0.002$, CCQ function score: $-0.431, P=0.001$ ) and the $\mathrm{BI}$ (CCQ total score: $r=-0.481$, $P<0.001$, CCQ function score: $-0.573, P<0.001)$. No correlation was found between the CCQ on admission (CCQ total score and CCQ separate domain scores) and lung function measured by the $\mathrm{FEV}_{1} \%$ of predicted. On admission, there was a moderate correlation between the mMRC dyspnoea scale and the CCQ total score and the CCQ function domain score. We found no significant correlation between the MMRC dyspnoea scale and the symptom domain of the CCQ. At discharge, we found a strong correlation between the CCQ total score and the CCQ function domain score and functional capacity measured by the 6MWT (CCQ total score: $r=-0.572, P<0.001)$ and the $\mathrm{BI}(\mathrm{CCQ}$ total score: $r=-0.539$, $P<0.001)$. To determine whether patients with an improvement in CCQ of more than the MCID are the same as those with clinically relevant functional improvements, we calculated the correlation coefficient between these variables. There was a moderate correlation between improvement in health status (CCQ-total

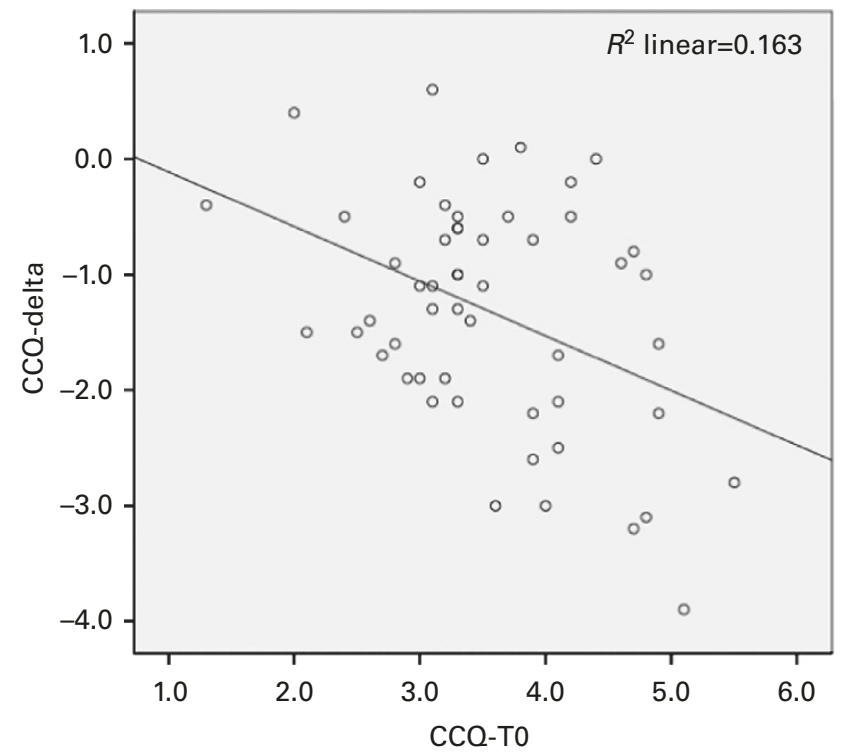

Figure 1. Scatterplot showing the relation between improvement in CCQ (CCQ-delta) and CCQ at baseline (CCQ-TO). Y-intercept $\left(B_{0}\right)=0.36^{\circ}$; gradient of regression line $\left(b_{1}\right)$ representing the change in outcome variable (CCQ-delta) associated with one-unit change in the predictor $(\mathrm{CCQ}-\mathrm{T} 0)=-0.473$.

score) and improvement in functional capacity (6MWT): $r=-0.432$, $P=0.002$. We found no significant correlation between improvement in CCQ and improvement in $\mathrm{BI}$ or between improvement in 6MWT and improvement in Bl. Of the subjects with an improvement in the CCQ score equal to or greater than the MCID, 67.7 and $73.9 \%$ also showed a clinically relevant improvement on the 6MWT and the $\mathrm{BI}$, respectively. Overall, $45.3 \%$ of the subjects showed clinically relevant improvements on all three outcome measurements (CCQ, 6MWT and $\mathrm{BI})$. We found no correlation between baseline lung function ( $\mathrm{FEV}_{1} \%$ of predicted) and improvement in health status or functional capacity.

\section{DISCUSSION}

Main findings

The first main finding of this study is that health status measured by the CCQ is severely impaired in this group of patients. Second, health status measured by the CCQ showed substantial and clinically relevant improvement during the PR programme; this improvement correlates well with improvement in functional capacity, indicating that the CCQ is sensitive to change in response to PR.

\section{Strengths and limitations of this study}

This is the first study that describes the course of health status as measured by the CCQ in patients with advanced COPD during a post-acute PR programme, with follow-up of almost all patients and few missing data at follow-up. However, this study warrants some limitations. Our population might be biased because we did not collect information on patients who were selected for the PR programme but were not motivated to participate. Patients who refused to participate were discharged from the hospital. The health status and functional capacity of our population may be even worse than that of the initial population that was indicated for PR in the hospital, because most patients who refused to participate were discharged home.

Furthermore, part of the improvement in health status measured by the CCQ might be caused by the 'in care effect' of patients participating in a study. PR is expected to improve functional 
Table 3. Correlations of CCQ score (total and subdomains) on admission (T0), discharge (T1) and change in CCQ scores (T1 - T0) with measurements of functional capacity, degree of dyspnoea and lung function

\begin{tabular}{|c|c|c|c|c|}
\hline$T$ & $B l^{a}$ & $6 M W T^{b}$ & $m M R C$ scale ${ }^{\mathrm{a}}$ & $F E V_{1}^{\mathrm{b}}$ \\
\hline \multicolumn{5}{|l|}{ TO } \\
\hline \multicolumn{5}{|c|}{ CCQ-total } \\
\hline$r$ & $-0.481^{c}$ & $-0.400^{c}$ & $0.392^{c}$ & -0.126 \\
\hline$P$ & $<0.001$ & 0.002 & 0.003 & 0.343 \\
\hline N & 60 & 57 & 55 & 59 \\
\hline \multicolumn{5}{|c|}{ CCQ-symptoms } \\
\hline$r$ & -0.209 & -0.167 & 0.117 & -0.072 \\
\hline$P$ & 0.126 & 0.235 & 0.414 & 0.605 \\
\hline N & 55 & 52 & 51 & 54 \\
\hline \multicolumn{5}{|c|}{ CCQ-function } \\
\hline$r$ & $-0.573^{c}$ & $-0.431^{c}$ & $0.437^{c}$ & -0.183 \\
\hline$P$ & $<0.001$ & 0.001 & 0.001 & 0.186 \\
\hline \multirow{2}{*}{\multicolumn{5}{|c|}{ CCQ-mental }} \\
\hline & & & & \\
\hline$r$ & -0.192 & -0.239 & 0.183 & -0.113 \\
\hline$P$ & 0.156 & 0.084 & 0.193 & 0.410 \\
\hline$N$ & 56 & 53 & 52 & 55 \\
\hline \multicolumn{5}{|l|}{$T 1$} \\
\hline \multicolumn{5}{|c|}{ CCQ-total } \\
\hline$r$ & $-0.572^{c}$ & $-0.539^{c}$ & & \\
\hline$P$ & $<0.001$ & $<0.001$ & & \\
\hline N & 53 & 51 & & \\
\hline \multicolumn{5}{|c|}{ CCQ-symptoms } \\
\hline$r$ & $-0.436^{c}$ & $-0.424^{c}$ & & \\
\hline$P$ & 0.002 & 0.003 & & \\
\hline$N$ & 48 & 47 & & \\
\hline \multicolumn{5}{|c|}{ CCQ-function } \\
\hline$r$ & $-0.579^{c}$ & $-0.503^{c}$ & & \\
\hline$P$ & $<0.001$ & $<0.001$ & & \\
\hline \multirow{2}{*}{\multicolumn{5}{|c|}{ CCQ-mental }} \\
\hline & & & & \\
\hline$r$ & -0.197 & $-0.297^{d}$ & & \\
\hline$P$ & 0.179 & 0.043 & & \\
\hline N & 48 & 47 & & \\
\hline \multicolumn{5}{|c|}{$T 1-T 0$} \\
\hline \multicolumn{5}{|c|}{ CCQ-total } \\
\hline$r$ & -0.099 & $-0.432^{c}$ & 0.058 & -0.223 \\
\hline$P$ & 0.481 & 0.002 & 0.694 & 0.113 \\
\hline N & 53 & 50 & 48 & 52 \\
\hline \multicolumn{5}{|c|}{ CCQ-symptoms } \\
\hline & -0.110 & $-0.449^{c}$ & 0.062 & -0.151 \\
\hline$P$ & 0.468 & 0.002 & 0.696 & 0.321 \\
\hline N & 46 & 44 & 42 & 45 \\
\hline \multicolumn{5}{|c|}{ CCQ-function } \\
\hline$r$ & -0.201 & $-0.313^{d}$ & 0.051 & $-0.304^{d}$ \\
\hline$P$ & 0.186 & 0.041 & 0.748 & 0.045 \\
\hline N & 45 & 43 & 42 & 44 \\
\hline \multicolumn{5}{|c|}{ CCQ-mental } \\
\hline$r$ & -0.067 & -0.268 & 0.007 & -0.069 \\
\hline$P$ & 0.568 & 0.78 & 0.963 & 0.650 \\
\hline$N$ & 46 & 44 & 42 & 45 \\
\hline
\end{tabular}

Abbreviations: $6 \mathrm{MWT}$, Six-Minute Walking Test; $\mathrm{BI}$, Barthel Index; FEV forced expiratory volume in $1 \mathrm{~s} ; \mathrm{mMRC}$, modified Medical Research Council. ${ }^{\text {a }}$ Spearman's correlation coefficient was calculated for non-normally distributed (BI) and interval (mMRC) measurements.

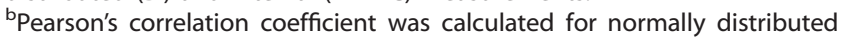
measurements (6MWT, FEV $\%$ pred).

cCorrelation is significant at the 0.01 level (two-tailed).

${ }^{\mathrm{d} C}$ Correlation is significant at the 0.05 level (two-tailed).

capacity, whereas improvement in the CCQ might partly be caused by participation in a study like the present one. Correlation between these two outcome measurements might therefore be overestimated. However, because of the observational design of the study and the fact that we solely measured a form of structured usual care, the 'in care effect' cannot be ruled out completely, but probably has limited influence on our results.

As the current study is an analysis of change between baseline and follow-up, a regression to the mean effect should be considered as a possible cause of observed change. The results of the linear regression analysis show that, when adjusted for baseline, improvement in CCQ reduces from -1.3 to -0.5 . This means that even after adjusting for regression to the mean there continues to be a significant and clinically relevant change.

To evaluate the use of the CCQ in this group of patients, comparison of the CCQ with another HRQoL instrument that is regularly used in this group of patients, namely, the Chronic Respiratory Questionnaire Self-Administered Standardized Format (CRQ-SAS), ${ }^{18}$ was initially included in the design of the study. However, during our study, compliance with the CRQ-SAS was very low, leading to very high rates of missing data $(\geqslant 50 \%)$, and we were therefore unable to present reliable results from the CRQSAS. Nevertheless, this is still an interesting result, as it also confirms that HRQoL instruments such as the CRQ are often timeconsuming and relatively difficult for patients to comprehend, leading to limited use, and usefulness, in daily practice. Compliance with the CCQ was, however, very good, leading to very few missing data ( $<2 \%$ at baseline; $8 \%$ at follow-up).

The last limitation is the fact that we did not have a control group, and although our results suggest that the CCQ is a responsive instrument for measuring change in health status following a post-acute PR programme in patients with advanced COPD, a randomised controlled trial would serve well to further confirm these findings.

Interpretation of findings in relation to previously published work Literature on health status as measured by the CCQ in patients with advanced COPD is scarce. The CCQ was originally developed and validated by van der Molen et $a l^{3}$ and has since been validated for the Italian ${ }^{19}$ and Greek language ${ }^{20}$ in patients with stable COPD. Compared with our results, data from these latter studies show lower CCQ scores (total scores and separate domain scores). This can be explained by the fact that our population suffered from a recent exacerbation and that exacerbations have a negative effect on health status. Recently, Kocks et al. ${ }^{21}$ reported data from two randomised controlled COPD exacerbation trials on the day-to-day course of patient-reported health status (as measured with the CCQ) during exacerbations. They reported results from 210 COPD patients admitted to hospital for an acute exacerbation (mean age 70.6 years, mean $\mathrm{FEV}_{1}: 37 \%$ of predicted); the CCQ total score on admission to the hospital was $3.3( \pm 0.93)$. Although this score is similar to our results, the time at which the CCQ score was measured is different, as we measured the CCQ on admission to the SNF. In the study by Kocks et al., ${ }^{21}$ the CCQ total score improved rapidly during hospital stay, with a mean score of 2.3 on day 7 . These results seem to confirm that our population indeed consisted of those patients who failed to recover during hospital stay.

Our results show a substantial and clinically relevant improvement in health status during the PR programme. This suggests that the CCQ is sensitive to change in response to PR in this group of patients. Literature on the responsiveness of the CCQ to interventions such as PR, or other forms of integrated care, is also scarce. The Picasso Bocholtz study ${ }^{6}$ evaluated the effect of Integrated Disease Management on health status as measured by the CCQ in 106 primary-care patients with mild to moderate COPD (mean age 64 years, mean $\mathrm{FEV}_{1} 63 \%$ of predicted). At the start of the study, the mean CCQ total score was 1.5, with an overall improvement of $-0.4(P=0.001)$ during follow-up. In the study by Damato et al., ${ }^{19}$ the CCQ showed sensitiveness to change in 46 patients undergoing an inpatient PR programme; the CCQ total score improved from 2.0 at baseline to 1.3 after PR $(P<0.001)$. Our data are in line with these studies, indicating that the CCQ is sensitive to change following interventions such as PR.

We found no correlation between CCQ total score at baseline and lung function ( $\mathrm{FEV}_{1} \%$ of predicted). This is in line with a growing body of evidence showing that traditional measurements of disease severity (such as lung function) do not correlate well with HRQoL or health status. ${ }^{1,2}$ However, our results differ from 
those of van der Molen et $a l_{.}^{3}$ and Damato et al. ${ }^{19}$ In both latter studies a significant correlation was found between the mean $\mathrm{FEV}_{1} \%$ of predicted and the mean CCQ total score (van der Molen et al.: $r=-0.38, P<0.01$; Damato et al.: $r=-0.57, P<0.01$ ). These correlation coefficients account for the total groups, including healthy smokers and subjects at risk. In COPD patients (GOLD stage 1-4), van der Molen et al. reported a correlation of $r=-0.49$ $(P<0.001)$. An explanation for these conflicting results can be that all our patients suffered from advanced COPD and thus differed substantially from those in the other two studies. With disease progression, health status deteriorates and is probably relatively less influenced by the degree of airflow limitation. During the PR programme, we found no correlation between baseline lung function ( $\mathrm{FEV}_{1} \%$ of predicted) and improvement in health status or functional capacity. This suggests that disease severity, as measured by the degree of airflow limitation, does not seem to predict which patients benefit most from the PR programme.

Implications for future research, policy and practice

In the present study, we evaluated the use of the CCQ in patients with advanced COPD admitted for post-acute PR. Considering our results, we recommend that the CCQ be used as a (primary) outcome measure in an experimental study design to evaluate the effect of post-acute (inpatient/outpatient) PR on health status in patients with advanced COPD. Our study also confirms that the CCQ is a practical and easy-to-use instrument for assessing health status, not only in research but also in daily practice. Our study was conducted with patients who were recruited after hospital admission for an acute exacerbation and admitted for inpatient PR. Thus, our patients were not treated in primary care during this study. However, in primary care, patients with advanced COPD are a growing group, with a huge burden of disease and in great need of better care. Therefore, research should also focus on the course of health status measured by the CCQ in patients with advanced COPD in primary care and the clinical use of the CCQ in elaborating tailormade medicine for this specific group of patients.

\section{Conclusions}

In patients with advanced COPD, health status measured by the CCQ improves after a post-acute PR programme. Moderate-tostrong correlations were found between the CCQ scores and functional capacity, showing that the CCQ correlates well with other important outcome measurements of PR. These results suggest that the CCQ is sensitive to change in response to PR in this group of patients.

\section{CONTRIBUTIONS}

EFvDvl designed the study, processed and analysed the collected data and wrote the manuscript. MS, KHGS, and NHC reviewed the manuscript. WPA participated in the design of the study, reviewed and helped writing the manuscript, and assisted with the statistical analysis. All authors have given final approval of the version to be published.

\section{COMPETING INTERESTS}

The authors declare no conflict of interest. NHC is an Associate editor of the PCRJ, but was not involved in the editorial review of, nor the decision to publish, this article.

\section{FUNDING}

EFvDvl is a part-time PhD student who is financially supported by Zorggroep Solis, Deventer, The Netherlands. Zorggroep Solis is the long-term- care facility at which the skilled nursing facility (SNF) that offers the geriatric rehabilitation programme for patients with advanced COPD is located.

\section{REFERENCES}

1 van der Molen T, Miravitlles M, Kocks JW. COPD management: role of symptom assessment in routine clinical practice. Int J Chron Obstruct Pulmon Dis 2013; 8: 461-471.

2 Sundh J, Janson C, Lisspers K, Montgomery S, Ställberg B. Clinical COPD Questionnaire score (CCQ) and mortality. Int J Chron Obstruct Pulmon Dis 2012; 7: 833-842.

3 van der Molen T, Willemse BW, Schokker S, ten Hacken NH, Postma DS, Juniper EF. Development, validity and responsiveness of the Clinical COPD Questionnaire. Health Qual Life Out 2003; 1: 13.

4 Kocks JW, Kerstjens HA, Snijders SL, de Vos B, Biermann JJ, van Hengel P et al. Health status in routine clinical practice: validity of the clinical COPD questionnaire at the individual patient level. Health Qual Life Out 2010; 8: 135.

5 Kocks JW, Asijee GM, Tsiligianni IG, Kerstjens HA, van der Molen T. Functional status measurement in COPD: a review of available methods and their feasibility in primary care. Prim Care Respir J 2011; 20: 269-275.

6 Kruis AL, van Adrichem J, Erkelens MR, Scheepers $\mathrm{H}$, In 't Veen $\mathrm{H}$, Muris JW et al. Sustained effects of integrated COPD management on health status and exercise capacity in primary care patients. Int J Chron Obstruct Pulmon Dis 2010; 5: 407-413.

7 Trappenburg JC, Touwen I, de Weert-van Oene GH, Bourbeau J, Monninkhof EM, Verheij TJ et al. Detecting exacerbations using the Clinical COPD Questionnaire. Health Qual Life Out 2010; 8: 102.

8 Celli BR, MacNee W, Agusti AATS/ERS Task Force. Standards for the diagnosis and treatment of patients with COPD: a summary of the ATS/ERS position paper. Eur Respir J 2004; 23: 932-946.

9 Holstege MS, Zekveld IG, Caljouw MA, Peerenboom PB, van Balen R, Gussekloo J et al. Relationship of patient volume and service concentration with outcome in geriatric rehabilitation. J Am Med Dir Assoc 2013; 14: 731-735.

10 Wells JL, Seabrook JA, Stolee P, Borrie MJ, Knoefel F. State of the art in geriatric rehabilitation. Part I: review of frailty and comprehensive geriatric assessment. Arch Phys Med Rehabil 2003; 84: 890-897.

11 Van Dam van Isselt EF, Groenewegen-Sipkema KH, Spruit-van Eijk M, Chavannes $\mathrm{NH}$, Achterberg WP. Geriatric rehabilitation for patients with advanced COPD: programme characteristics and case studies. Int J Palliat Nurs 2013; 19: 141-146.

12 Kocks JW, Tuinenga MG, Uil SM, van den Berg JW, Ståhl E, van der Molen T et al. Health status measurement in COPD: the minimal clinically important difference of the clinical COPD questionnaire. Respir Res 2006; 7: 62.

13 Collin C, Wade DT, Davies S, Horne V. The Barthel ADL index: a reliability study. Int Disabil Stud 1988; 10: 61-63.

14 Hsieh YW, Wang CH, Wu SC, Chen PC, Sheu CF, Hsieh CL. Establishing the minimal clinically important difference of the Barthel Index in stroke patients. Neurorehabil Neural Repair 2007; 21: 233-238.

15 ATS Committee on Proficiency Standards for Clinical Pulmonary Function Laboratories. ATS statement. guidelines for the six-minute walking test. Am J Respir Crit Care Med 2002; 166: 111-117.

16 Puhan MA, Chandra D, Mosenifar Z, Ries A, Make B, Hansel NN et al. The minimal important difference of exercise tests in severe COPD. 1. Eur Respir J 2011; 37: 784-790.

17 Puhan MA, Mador MJ, Held U, Goldstein R, Guyatt GH, Schünemann HJ. Interpretation of treatment changes in 6-minute walk distance in patients with COPD. Eur Respir J 2008; 32: 637-643.

18 Schünemann HJ, Griffith L, Jaeschke R, Goldstein R, Stubbing D, Austin P et al. A comparison of the original chronic respiratory questionnaire with a standardized version. Chest 2003; 124: 1421-1429.

19 Damato S, Bonatti C, Frigo V, Pappagallo S, Raccanelli R, Rampoldi C et al. Validation of the Clinical COPD Questionnaire in Italian language. Health Qual Life Out 2005; 3: 9.

20 Papadopoulos G, Vardavas Cl, Limperi M, Linardis A, Georgoudis G, Behrakis P. Smoking cessation can improve quality of life among COPD patients: validation of the clinical COPD questionnaire into Greek. BMC Pulm Med 2011; 11: 13.

21 Kocks JW, van den Berg JW, Kerstjens HA, Uil SM, Vonk JM, de Jong YP et al. Dayto-day measurement of patient-reported outcomes in exacerbations of chronic obstructive pulmonary disease. Int J Chron Obstruct Pulmon Dis 2013; 8: 273-286.

\footnotetext{
(c) (1) $\Theta$ This work is licensed under a Creative Commons AttributionNonCommercial-NoDerivatives 4.0 International License. The images or other third party material in this article are included in the article's Creative Commons license, unless indicated otherwise in the credit line; if the material is not included under the Creative Commons license, users will need to obtain permission from the license holder to reproduce the material. To view a copy of this license, visit http:// creativecommons.org/licenses/by-nc-nd/4.0/
} 